\title{
Nuclear matter description based on quark structure and pion exchange
}

\author{
R. Huguet, J.C. Caillon* and J. Labarsouque \\ Centre d'Etudes Nucléaires de Bordeaux-Gradignan, CNRS-IN2P3, \\ Université Bordeaux 1, Le Haut-Vigneau, 33170 Gradignan Cedex, France. \\ E-mail: caillondcenbg.in2p3.fr
}

\begin{abstract}
We have investigated[1] the possibility to describe infinite and symmetric nuclear matter in an approach constrained by quantum chromodynamics (QCD). More precisely, we have mapped the nucleon self-energies of a point coupling relativistic mean-field model on self-energies obtained in effective theories of QCD. We have determined the contributions to in-medium nucleon selfenergy by separating the short range part, driven principally by the quark structure of the nucleon, from the long range part, dictated by pion dynamics.
\end{abstract}

We have taken the nucleon structure into account in a simple quark-diquark picture in a NambuJona-Lasinio model[2], which is chirally invariant and reproduces the spontaneous chiral symmetry breaking. The quark-diquark picture generates large attractive scalar and repulsive vector self-energies in the medium, with an average ratio at saturation density of $\Sigma_{S}^{0} / \Sigma_{V}^{0} \sim-2$.

On the other hand, the long range part has been determined by including the one pion and iterated one pion exchange described in the framework of in-medium chiral perturbation theory[3]. The self-energies obtained are found to be approximately $130 \mathrm{MeV}$ at saturation for scalar and vector channels.

A saturation point with too low density is obtained without any free parameter to fine tune (for a given constituent quark mass value). The description of nuclear matter saturation properties (saturation density $\rho_{\text {sat }}$, binding energy $\mathscr{E}_{B}\left(\rho_{\text {sat }}\right)$, effective nucleon mass $M_{N}^{*}\left(\rho_{\text {sat }}\right)$ and incompressibility modulus $K\left(\rho_{\text {sat }}\right)$ ) is improved by introducing a correction term to the self-energy, linear in the density, which could be interpreted as a correction to the quark-diquark picture approximations. This term is found to be relatively small, and we get a reasonable description of nuclear saturation considering that, for a given quark mass value, we have only one free parameter $\delta G^{0}$.

8th Conference Quark Confinement and the Hadron Spectrum

September 1-6 2008

Mainz, Germany

${ }^{*}$ Speaker. 


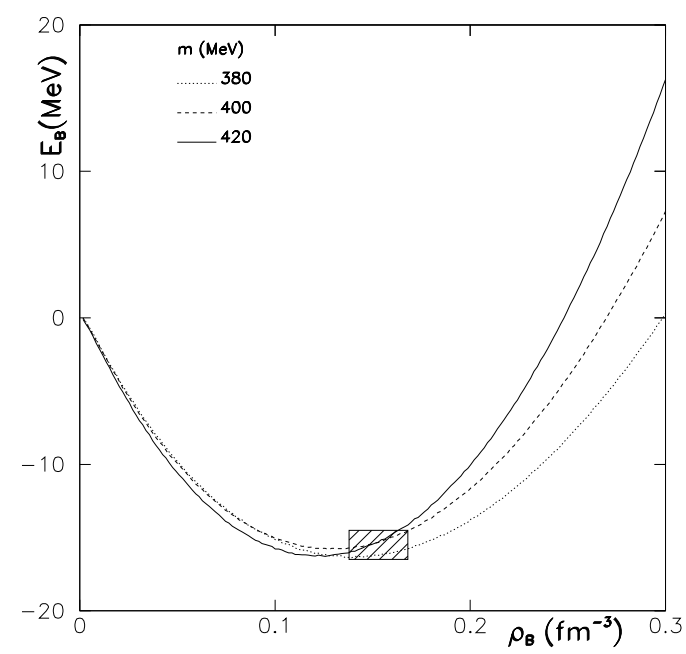

Figure 1: Binding energy per nucleon versus baryonic density for three constituent quark mass values: $m=380 \mathrm{MeV}$ (dotted line), $m=400 \mathrm{MeV}$ (dashed line), $m=420 \mathrm{MeV}$ (solid line).

The results[1] for different quark mass values $m$ are listed in Table 1, and the binding energy versus baryonic density is represented on Fig. 1 for three quark mass values (for $m<370 \mathrm{MeV}$, the nucleon is not bound and for $m>440 \mathrm{MeV}$ the scalar self-energy of the nucleon is too much attractive).

\begin{tabular}{cccccc}
\hline \hline$m(\mathrm{MeV})$ & $\delta G^{0}\left(\mathrm{fm}^{2}\right)$ & $\rho_{\text {sat }}\left(\mathrm{fm}^{-3}\right)$ & $\mathscr{E}_{B}(\mathrm{MeV})$ & $M_{N}^{*} / M_{N}$ & $K(\mathrm{MeV})$ \\
\hline 380 & -2.9 & 0.134 & -16.3 & 0.81 & 249 \\
390 & -2.8 & 0.134 & -16.2 & 0.82 & 245 \\
400 & -2.1 & 0.128 & -16.4 & 0.77 & 260 \\
410 & -1.7 & 0.122 & -16.3 & 0.75 & 267 \\
420 & -1.4 & 0.12 & -16.3 & 0.73 & 274 \\
\hline \hline
\end{tabular}

Table 1: $\delta G^{0}$ values and saturation properties of nuclear matter for different quark mass values (see text).

It is clear from Fig. 1 that the binding energy is almost independent of the constituent quark mass value for densities around and below the saturation point. Although there is only one free parameter, the saturation properties are reasonable for any of the quark mass values considered (however, the saturation density is somewhat too low and the effective nucleon mass too high). These results indicate that, by refining the quark-diquark description of the nucleon, the model could be noticeably improved.

\section{References}

[1] R. Huguet, J.C. Caillon and J. Labarsouque, Nucl. Phys. A809 (2008) 189;

[2] Y. Nambu and G. Jona-Lasinio, Phys. Rev. 122 (1961) 345; Phys. Rev. 124 (1961) 246

[3] N. Kaiser, S. Fritsch and W. Weise, Nucl. Phys. A697. (2002) 255 